\title{
Spectroscopy of B-type emission-line stars with compact dusty envelopes: HD 85567, Hen 3-140, and Hen 3-1398
}

\author{
A. S. Miroshnichenko ${ }^{1,2}$, H. Levato ${ }^{3}$, K. S. Bjorkman ${ }^{1}$, and M. Grosso ${ }^{3}$ \\ 1 Ritter Observatory, Dept. of Physics \& Astronomy, University of Toledo, Toledo, OH 43606, USA \\ 2 Central Astronomical Observatory of the Russian Academy of Sciences at Pulkovo, 196140 Saint-Petersburg, \\ Russia \\ 3 Complejo Astronómico El Leoncito (CASLEO), Casilla de Correo 467, 5400 San Juan, Argentina \\ Received 6 February 2000 / Accepted 8 March 2001
}

\begin{abstract}
We report the results of our spectroscopic observations (3600-5900 $\AA, R \simeq 16000$ and 5750-8750 $\AA$, $R \simeq 11000$ ) of three southern emission-line B-type stars associated with IRAS sources, HD 85567, Hen 3-140, and Hen 3-1398, whose nature and evolutionary state are not well-established yet. We also obtained new multicolour photometry of HD $85567\left(U B V(R I)_{\mathrm{c}} J H K L\right)$ and Hen 3-140 (JHKLM). A large near-IR excess is found in Hen 3140 for the first time. All three objects show double-peaked Fe II lines in emission. The Balmer emission lines are double-peaked in HD 85567 and Hen 3-140, while the He I lines are found in absorption. Hen 3-1398 displays triple-peaked Balmer lines as well as single-peaked He I and He II lines in emission. Fundamental parameters of the stars are estimated on the basis of both photometric and spectroscopic data. The whole set of their properties resemble that of a recently defined group of Be stars with warm dust (Sheikina et al. 2000).
\end{abstract}

Key words. stars: emission-line - stars: circumstellar matter - stars: individual (HD 85567, Hen 3-140, Hen 3-1398)

\section{Introduction}

A large number of emission-line B-type stars were detected by the IRAS satellite because of the presence of infrared (IR) excesses in their spectral energy distributions (SED). These excesses are due to radiation of circumstellar gas and dust. Studies of their shapes and strengths help to constrain properties of the circumstellar matter and, in many cases and in combination with spectroscopic studies, the evolutionary state of the star. For example, Be stars with free-free IR excesses are main sequence objects, those with much stronger IR excesses due to dust particles in a wide range of temperatures and moderate luminosities are pre-main-sequence ones, while high-luminosity Betype stars with dusty IR excesses are usually post-mainsequence LBV stars or $\mathrm{B}[\mathrm{e}]$ supergiants. However, it is difficult to form conclusions on the nature and evolutionary state of objects with dusty IR excesses, even with extensive observational data in hand. This was demonstrated by Thé et al. (1994), who compiled a catalogue of $287 \mathrm{such}$ objects, properties of only about $50 \%$ of which are thought to be well understood.

One of the examples of poorly-understood groups of early-type stars with IR excesses is the $\mathrm{B}[\mathrm{e}]$ stars selected by Allen \& Swings (1976), many of which are still unclassified (Lamers et al. 1998). Some of these

\footnotetext{
Send offprint requests to: A. S. Miroshnichenko,

e-mail: anatoly@physics.utoledo.edu
}

unclassified $B[e]$ stars show large near-IR excesses and a steep decrease of IR fluxes longward of $12 \mu \mathrm{m}$ (e.g., Sheikina et al. 2000). Only about 10 such objects are contained in the original list of Allen \& Swings (1976). However, the IRAS survey revealed more poorly studied early-type stars with the mentioned IR properties; these stars are the focus of our investigation. Recently we completed an extensive study of 2 new members of this group, AS 78 and MWC 657 (Miroshnichenko et al. 2000), and suggested that they might be binaries. Here we report the results of a study of three southern emission-line objects (HD 85567, Hen 3-140, and Hen 3-1398), whose properties turned out to be similar to those of the objects from Sheikina et al. (2000). For the sake of brevity, hereafter we will refer to entire group as Be stars with warm dust.

The objects of this study, which are listed in the catalogue of The et al. (1994), were selected on the basis of their IRAS colours (see Fig. 1). They are moderately reddened in the visual region and are not associated with nebulosity. Basic information about their positions in the sky, previously published spectral types, and brightnesses is presented in Table 1. We obtained high-resolution optical spectroscopy of HD 85567, Hen 3-140, and Hen 3-1398, expecting them to show peculiar spectra similar to those of other stars from this group. As we will show below this did turn out to be the case. The spectroscopic results, supplemented by additionally obtained photometry and other information from the literature, allowed us to 
Table 1. Initial information about the objects

\begin{tabular}{rccrlrrr}
\hline Name & RA & Dec. & $V$ & Sp.T. & $F_{12}$ & $F_{25}$ & $F_{60}$ \\
\hline Hen 3-140 & $08^{\mathrm{h}} 14^{\mathrm{m}} 20^{\mathrm{s}}$ & $-50^{\circ} 09^{\prime} 46^{\prime \prime}$ & 10.11 & B8e & 5.4 & 4.7 & 1.2 \\
HD 85567 & $09^{\mathrm{h}} 50^{\mathrm{m}} 29^{\mathrm{s}}$ & $-60^{\circ} 58^{\prime} 03^{\prime \prime}$ & 8.55 & B5 Vne & 6.4 & 5.8 & 1.4 \\
Hen 3-1398 & $17^{\mathrm{h}} 24^{\mathrm{m}} 45^{\mathrm{s}}$ & $-38^{\circ} 43^{\prime} 52^{\prime \prime}$ & 10.57 & Oe & 21.2 & 12.1 & 3.2 \\
\hline
\end{tabular}

Coordinates on epoch 2000.0 are given in Cols. 2 and 3, the $V$-band brightness and spectral type in Cols. 4 and 5 , non colour corrected IRAS fluxes in Janskys in Cols. 6-8.

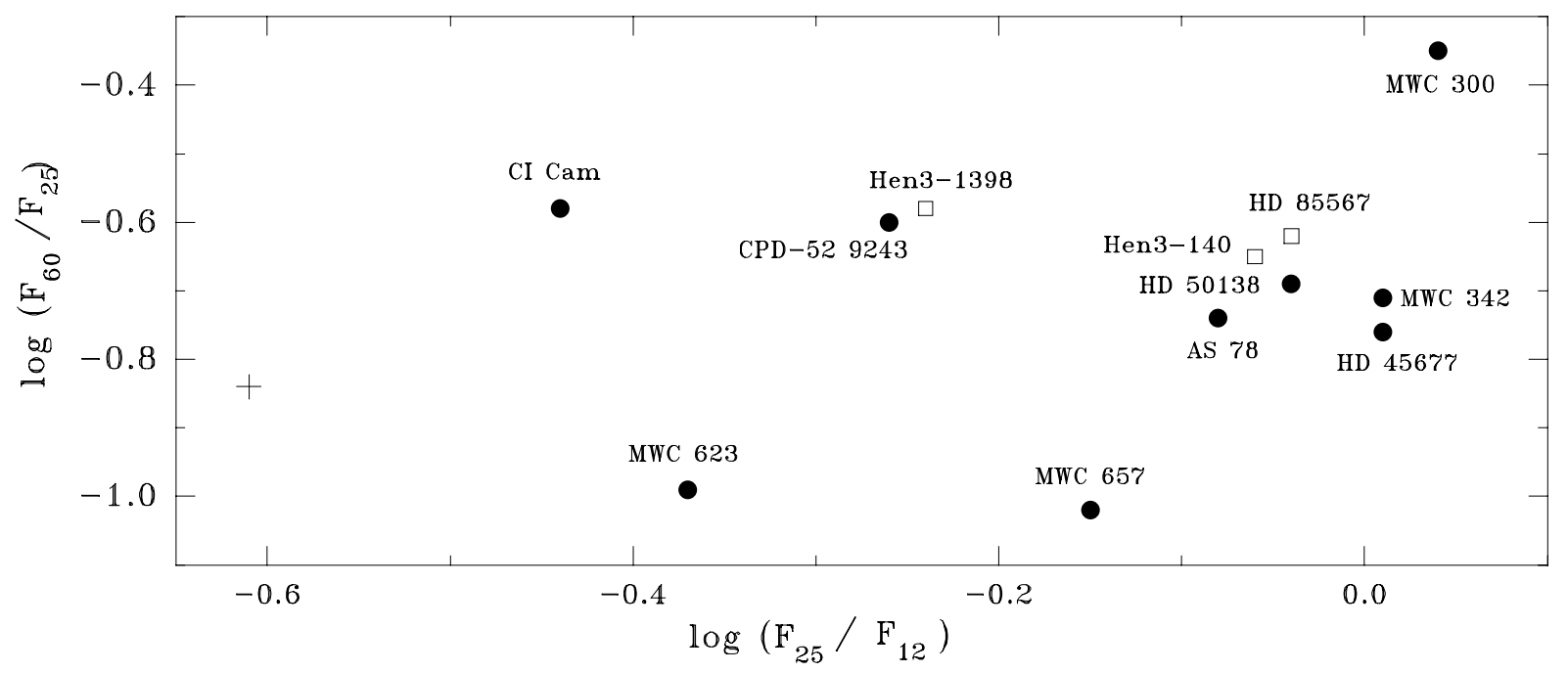

Fig. 1. The IRAS colour-colour diagram. The cross denotes the photospheric colour-indices, which show a very weak dependence on stellar temperature in this wavelength region. Filled circles represent positions of Be stars with warm dust from Sheikina et al. (2000)

estimate fundamental parameters of the stars and discuss their nature and evolutionary state. This paper continues the series of our papers on Be stars with warm dust (the most recent papers are Miroshnichenko \& Corporon 1999; Sheikina et al. 2000; Miroshnichenko et al. 2000). It is intended to introduce the complex properties of these three objects and outline a program for future study.

\section{Observations}

Spectroscopic observations were obtained on 2000 February 5 (HD 85567 and Hen 3-140, 3800-5900 ̊), April 22 (Hen 3-1398, 3600-5400 A), and June 26 (HD 85567, 5700-8750 $\AA$ ) at the 2.1-meter telescope of the Complejo Astronomico El Leoncito (CASLEO, Argentina). We used a REOSC echelle-spectrograph with an echelle grating of 79 lines $\mathrm{mm}^{-1}$ and a cross disperser of $400 \mathrm{lmm}^{-1}$ centered around $4500 \AA$. The REOSC is on loan from the Institute $\mathrm{d}^{\prime}$ Astrophysique de Liège, Belgium. A 2-pixel slit was used, and the detector was a TEK matrix $1024 \times 1024$ with $24-\mu$ m pixels. The 2 -pixel resolution in the blue region is about $0.28 \AA$, while it is $0.62 \AA$ in the red region. Three exposures for HD 85567 (5 min each) and Hen 3-140 (20 min each) were obtained on 2000 February 5 , one 20-min exposure was obtained for Hen 3-1398 on April 22, and one exposure for HD 85567 on 2000 June 26. No dark exposures were taken, as the dark current of the detector is only $0.4 \mathrm{e}^{-}$per hour at $-110 \mathrm{C}$. The spectra were reduced and normalized to the continuum using standard techniques in $\operatorname{IRAF}^{1}$.

The optical and near-IR broadband photometric observations of HD 85567 were obtained quasi-simultaneously on 1997 February 26 and March 2 at the South-African Astronomical Observatory (SAAO). Additional near-IR data were obtained earlier by $T$. Lloyd Evans. The 0.75-meter telescope with a single-element InSb photometer was used in the near-IR, while the 0.5-meter telescope with a GaAs photometer was used in the optical region. The observations of Hen 3-140 were obtained on 2000 February 25 at Mauna Kea with the 3-meter NASA Infrared Telescope Facility (IRTF) and a single-element gallium-doped germanium bolometer. Our near-IR data are presented in Table 2. The accuracy is about $0.01 \mathrm{mag}$ and $0.03 \mathrm{mag}$ for the SAAO optical and IR data, respectively, and $0.05 \mathrm{mag}$ for the IRTF data. A number of photometric standard stars were observed during the same nights at both observatories for calibration.

\footnotetext{
${ }^{1}$ IRAF is distributed by the National Optical Astronomy Observatories, which are operated by the Association of Universities for Research in Astronomy, Inc., under contract with the National Science Foundation.
} 
Table 2. New photometry of HD 85567 and Hen 3-140

\begin{tabular}{lcccccc}
\hline Name & JD & $U$ & $B$ & $V$ & $R_{\mathrm{c}}$ & $I_{\mathrm{c}}$ \\
& $2400000+$ & & & & & \\
\hline HD 85567 & 50506.40 & 8.16 & 8.70 & 8.57 & 8.36 & 8.12 \\
& 50510.40 & 8.18 & 8.71 & 8.57 & 8.36 & 8.11 \\
\hline Name & JD & $J$ & $H$ & $K$ & $L$ & $M$ \\
& $2400000+$ & & & & & \\
\hline HD 85567 & 48820.21 & 7.54 & 6.62 & 5.70 & 4.56 & \\
& 50506.46 & 7.55 & 6.63 & 5.70 & 4.56 & \\
& 50510.44 & 7.55 & 6.62 & 5.69 & 4.55 & \\
Hen 3-140 & 49120.25 & 9.06 & 8.30 & 7.38 & 5.93 & \\
& 51599.85 & & & 7.45 & 6.25 & 4.86 \\
\hline
\end{tabular}

\section{Previous studies}

\subsection{Hen $3-140$}

Henize (1976), who discovered the object as an emissionline star, gave no estimate of its spectral type. The only reference to the spectral type, B8e, is listed in Thé et al. (1994) with no comments about its source. No photoelectric photometry of Hen 3-140 has been published so far. Yudin \& Evans (1998) measured optical polarization of Hen 3-140 which turned out to be $0.8 \pm 0.4 \%, 1.1 \pm 0.3 \%$, and $0.6 \pm 0.2 \%$ in the $U, B$, and $V$-band, respectively. These authors found a wavelength dependence of the polarization angle $\left(\Theta_{U}=132^{\circ} \pm 16^{\circ}, \Theta_{B}=116^{\circ} \pm 9^{\circ}, \Theta_{V}=\right.$ $\left.94^{\circ} \pm 5^{\circ}\right)$ and suggested that the polarization is of circumstellar origin.

\section{2. $H D 85567$}

HD 85567 was classified as B5 vne by Houk \& Cowley (1975) and as B8 v:ne by Garrison et al. (1977) on the basis of objective prism spectra. $U B V$ photometry was obtained by Klare \& Neckel (1977) and Shild et al. (1983), while multicolour data (in the Geneva photometric system and $J H K L^{\prime} M$ bands) were published by Malfait et al. (1998). The results are very close to each other and to our data, indicating no significant variability exceeding the observational errors. HD 85567 was also observed in the UV region by the TD1 and IUE satellites.

Klare \& Neckel (1977) and Yudin \& Evans (1998) measured optical polarization of HD 85567, finding values of $0.2 \pm 0.1 \%$ at $\Theta=113^{\circ} \pm 15^{\circ}$ and $0.45 \pm 0.05 \%$ at $\Theta=105^{\circ} \pm 5^{\circ}$, respectively. These results seem to be consistent with each other as Klare \& Neckel (1977) apparently used a different spectral region (probably bluer) than Yudin \& Evans (1998), who observed in the $V$-band.

Oudmaijer et al. (1992) identified HD 85567 with an IRAS point source and suggested that it is a pre-mainsequence Herbig Ae/Be star. Malfait et al. (1998) fitted its SED with a two-component optically-thin dusty envelope model. One of the components is located between 100 and 2000 stellar radii $\left(R_{*}\right)$ and contains only hot dust, while the other one is located outside of $3500 R_{*}$ and contains cool dust. The apparent dip in the SED between 6 and $10 \mu \mathrm{m}$ was interpreted as a physical hole in the dust distribution, caused by the break-up of the optically thin dusty disk (e.g., Lada \& Adams 1992).

A lower limit on the distance towards HD $85567, D \geq$ 480 pc, was determined by the HIPPARCOS (ESO 1997) parallax measurement. van den Ancker et al. (1998) used this distance to estimate the star's luminosity which, under the assumptions of the spectral type B7/8 and interstellar extinction $A_{V}=0.81 \mathrm{mag}$, turned out to be $\log L_{\mathrm{bol}} / L_{\odot} \geq 2.54$. The corresponding position in the Hertzsprung-Russell diagram (HRD) is far $(\geq 0.5 \mathrm{dex})$ from the zero-age main-sequence (ZAMS) and close to the birthline for Herbig Ae/Be stars (Palla \& Stahler 1993), which may indicate that the star is very young.

\subsection{Hen $3-1398$}

Hen 3-1398 was discovered by Henize (1976), who estimated its spectral type as B, while Thé et al. (1994) listed it as Oe. $U B V$ photometry was obtained by Kozok (1985a) and Drilling (1991), and their values are very close to each other $(V=10.61$ and $10.53, B-V=0.76$ and 0.71 , $U-B=-0.32$ and -0.30 , respectively). Using the photometric data, Kozok (1985b) estimated the interstellar colour-excess $E_{B-V}=1.08 \mathrm{mag}$, the absolute visual magnitude $M_{V}=-3.66 \mathrm{mag}$, and the distance toward the star $D=1.58 \mathrm{kpc}$, assuming some average relationship between stellar intrinsic colours and luminosities. This author admitted that the uncertainty in distance moduli for early B-type stars calculated using his method may be as large as 0.8 mag. The interstellar extinction law in the direction of Hen 3-1398 given by Neckel \& Klare (1980) is not well constrained, as they had data for only two stars in the object's vicinity. Thus, the information about the fundamental parameters and distance of Hen 3-1398 needs to be revised.

The $J H K L^{\prime} M$ photometry was obtained by Guglielmo et al. (1993) and revealed a large nearIR excess due to warm dust. This is supported by the IRAS LRS spectrum, which contains a weak silicate $10 \mu \mathrm{m}$ emission feature (Volk et al. 1991). Recently the object was detected by the MSX satellite (source MSX5C-G349.4206-01.6561, Egan et al. 1999) with fluxes comparable to measured by IRAS. No polarimetric or spectroscopic measurements of Hen 3-1398 have been reported in the literature.

Thus, it follows from the above literature analysis that HD 85567 and Hen 3-1398 do not show noticeable photometric variations, their SEDs have been measured in a wide spectral range, but analysis of only HD 85567 has been attempted. The absence of spectroscopic data (and photometric data for Hen 3-140) hampered further studies of the objects, which show rather peculiar (for early-type stars) SEDs in the IRAS wavelength region. 
Table 3. Lines identified in the spectra of HD 85567 and Hen 3-140

\begin{tabular}{|c|c|c|c|c|c|c|c|c|c|}
\hline \multirow[t]{2}{*}{ Line } & \multirow[t]{2}{*}{$\lambda_{\text {lab }}$} & \multicolumn{4}{|c|}{ HD 85567} & \multicolumn{4}{|c|}{ Hen 3-140 } \\
\hline & & $I / I_{\mathrm{c}}$ & $R V$ & $E W$ & Rem. & $I / I_{\mathrm{c}}$ & $R V$ & $E W$ & Rem. \\
\hline Ca II H (1) & 3933.66 & $0.60 / 1.09$ & $+2^{\mathrm{b}}$ & & & 0.34 & +36 & 0.79 & \\
\hline Ca II K (1) & 3968.47 & & & & blend & 0.46 & $+32:$ & & blend \\
\hline $\mathrm{H} \epsilon^{\mathrm{a}}$ & 3970.07 & & & & comp. & 0.16 & $+19:$ & 2.6 & $+\mathrm{p}$ \\
\hline He I (18) & 4026.18 & 0.81 & -3 & 0.30 & & 0.86 & +34 & 0.31 & \\
\hline $\mathrm{H} \delta$ & 4101.74 & $0.44 / 0.97$ & & & comp. & 0.18 & +17 & 3.1 & em.comp. \\
\hline He I (16) & 4120.81 & $0.95:$ & & & weak & $0.95:$ & & 0.02: & weak \\
\hline Fe II $(28)$ & 4122.64 & $0.96:$ & & & weak & & & & \\
\hline Si II (3) & 4128.05 & 0.88 & $+4:$ & $0.1:$ & noisy & 0.93: & & & noisy \\
\hline Si II (3) & 4130.88 & 0.86 & & 0.1 : & noisy & 0.93: & & & noisy \\
\hline He I (16) & 4143.76 & 0.83 & -18 & $0.2:$ & noisy & 0.77 & $+45:$ & $0.24:$ & \\
\hline Fe II $(27)$ & 4173.45 & & & & & 0.95 & $+31:$ & 0.02: & \\
\hline Fe II $(27)$ & 4233.17 & 1.09 & & & dp: & 0.94 & +39 & 0.03 & \\
\hline Fe II $(32)$ & 4278.17 & & & & & 0.89 & +16 & 0.07 & \\
\hline Fe II $(27)$ & 4303.17 & 0.92 & $-21:$ & 0.02: & blend & & & & \\
\hline $\mathrm{H} \gamma$ & 4340.47 & $0.41 / 1.34$ & & & comp. & 0.19 & +19 & 2.1 & \\
\hline Fe II $(27)$ & 4351.76 & 1.07 & & & $\mathrm{dp}:$ & & & & \\
\hline He I (51) & 4387.93 & 0.90 & +1 & 0.13 & & 0.96 & +21 & 0.05 & \\
\hline He I (14) & 4471.48 & 0.88 & +3 & 0.19 & & 0.84 & +40 & 0.37 & \\
\hline Mg II (4) & 4481.13 & 0.82 & -2 & 0.13 & & 0.81 & +35 & 0.37 & \\
\hline Fe II (37) & 4582.84 & 1.07 & & 0.25 & dp: & 0.96 : & & 0.05: & weak \\
\hline He I (12) & 4713.14 & 0.94 & +4 & 0.05 & & 0.96 & +33 & 0.05 & \\
\hline $\mathrm{H} \beta$ & 4861.33 & $0.39 / 2.69$ & & & P Cyg & $0.32 / 2.15$ & +16 & & $\mathrm{dp}$ \\
\hline Fe II $(42)$ & 4923.92 & 1.11 & & 0.23 & $\mathrm{dp}$ & & & & \\
\hline Fe II $(42)$ & 5018.43 & 1.17 & & 0.70 & $\mathrm{dp}$ & $0.94 / 1.05$ & +28 & & dp: \\
\hline Si II (14) & 5056.02 & 0.91 & -10 & 0.05 & & & & & \\
\hline Fe II $(42)$ & 5169.03 & 1.18 & & 1.20 & $\mathrm{dp}$ & 0.84 & +33 & 0.08 & \\
\hline Fe II (49) & 5234.62 & 1.04 & & & weak & & & & \\
\hline Fe II (49) & 5316.61 & 1.08 & & & $\mathrm{dp}:$ & & & & \\
\hline$\left[\begin{array}{ll}\mathrm{O} & \mathrm{I}\end{array}\right](3 \mathrm{~F})$ & 5577.35 & 1.08 & +17 & 0.07 & tell.: & 1.32 & +3 & 0.07 & tell.: \\
\hline DIB 5780 & 5780.41 & 0.95 & +10 & 0.08 & & 0.94 & +14 & 0.13 & dp: \\
\hline DIB 5797 & 5797.03 & 0.96 & $+15:$ & 0.02 & noisy & 0.96 & & 0.05 & noisy \\
\hline HeI $(2)$ & 5875.65 & 0.87 & -5 & 0.12 & & 0.80 & +67 & 0.71 & \\
\hline NaI (1) & 5889.95 & $0.59 / 1.09$ & +4 & $0.24^{\mathrm{b}}$ & & 0.30 & +19 & 0.43 & \\
\hline $\mathrm{NaI}(1)$ & 5895.92 & $0.76 / 1.05$ & +5 & $0.15^{\mathrm{b}}$ & & 0.45 & +20 & 0.34 & \\
\hline
\end{tabular}

a - Blend with Ca II K (1).

b - Only for the absorption component; displays a double-peaked emission.

A colon after the figure indicates an uncertain value; $\mathrm{dp}$ - a double-peaked profile; tell.: - possible telluric contribution; P Cyg - P Cyg-type profile; comp. - a multicomponent profile; + p - refers to the previous line; em. comp. - the profile contains an emission component.

Table 4. Adopted parameters of the objects

\begin{tabular}{lcccccc}
\hline Name & $\begin{array}{c}T_{\text {eff }} \\
\mathrm{K}\end{array}$ & $\log g$ & $\begin{array}{c}v \sin i \\
\mathrm{~km} \mathrm{~s}^{-1}\end{array}$ & $\begin{array}{c}E_{B-V} \\
\mathrm{mag}\end{array}$ & $\begin{array}{c}D \\
\mathrm{kpc}\end{array}$ & $\log \left(L_{\mathrm{bol}} / L_{\odot}\right)$ \\
\hline Hen 3-140 & 12000 & 4.0 & 70 & 0.3 & $2.0 \pm 0.5$ & $3.1 \pm 0.2$ \\
HD 85567 & 19000 & 3.5 & 50 & 0.4 & $1.5 \pm 0.5$ & $4.0 \pm 0.3$ \\
Hen 3-1398 & 30000 & 4.0 & 50 & 1.1 & $3.3 \pm 0.4$ & $5.3 \pm 0.2$ \\
\hline
\end{tabular}



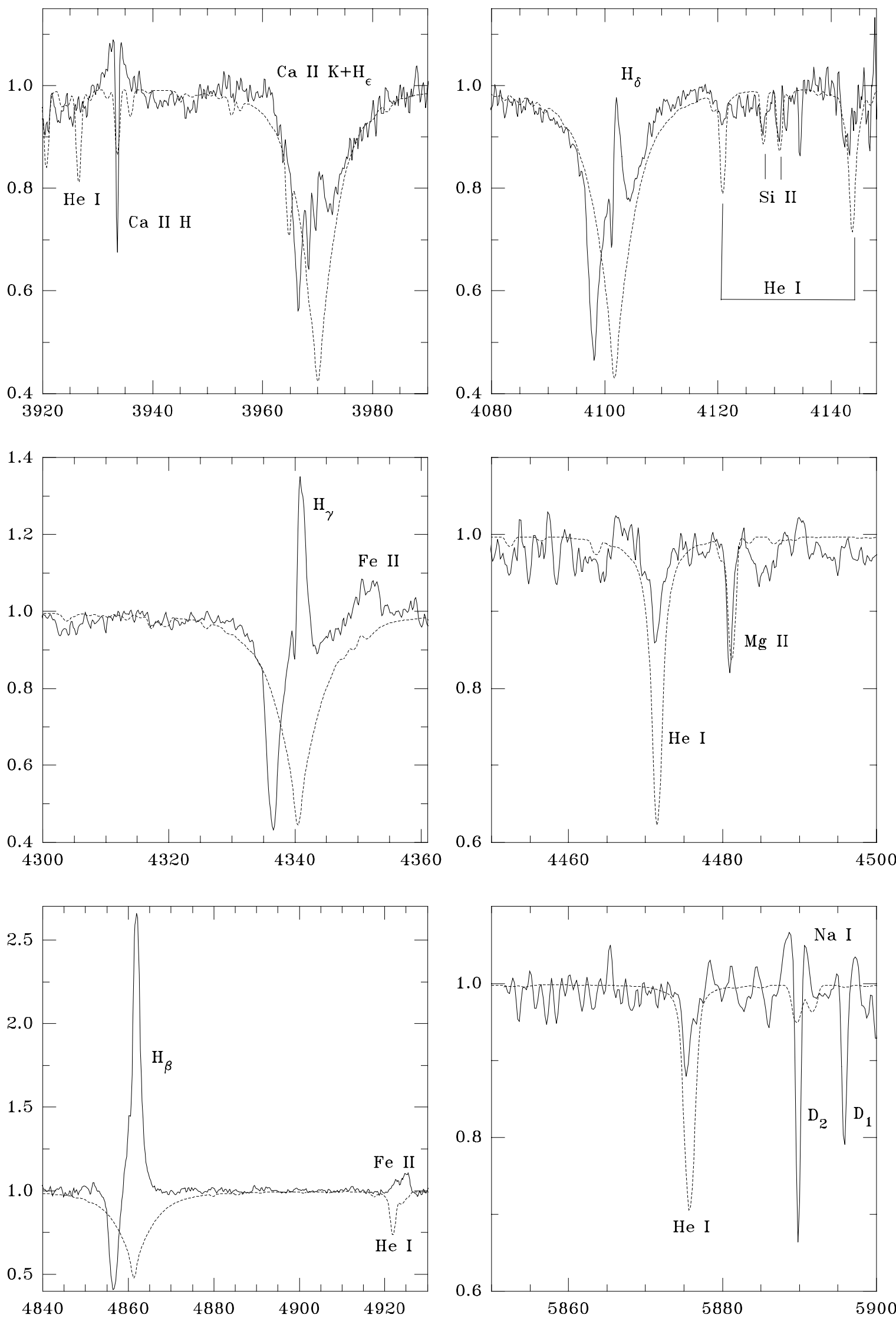

Fig. 2. Parts of the spectrum of HD 85567. The stellar spectrum is shown by solid lines, while the theoretical one (for $T_{\text {eff }}=$ $19000 \mathrm{~K}, \log g=3.5$, and $v \sin i=50 \mathrm{~km} \mathrm{~s}^{-1}$ ) is shown by dashed lines. The intensity is normalized to the underlying continuum, the wavelength scale is given in $\AA$ 

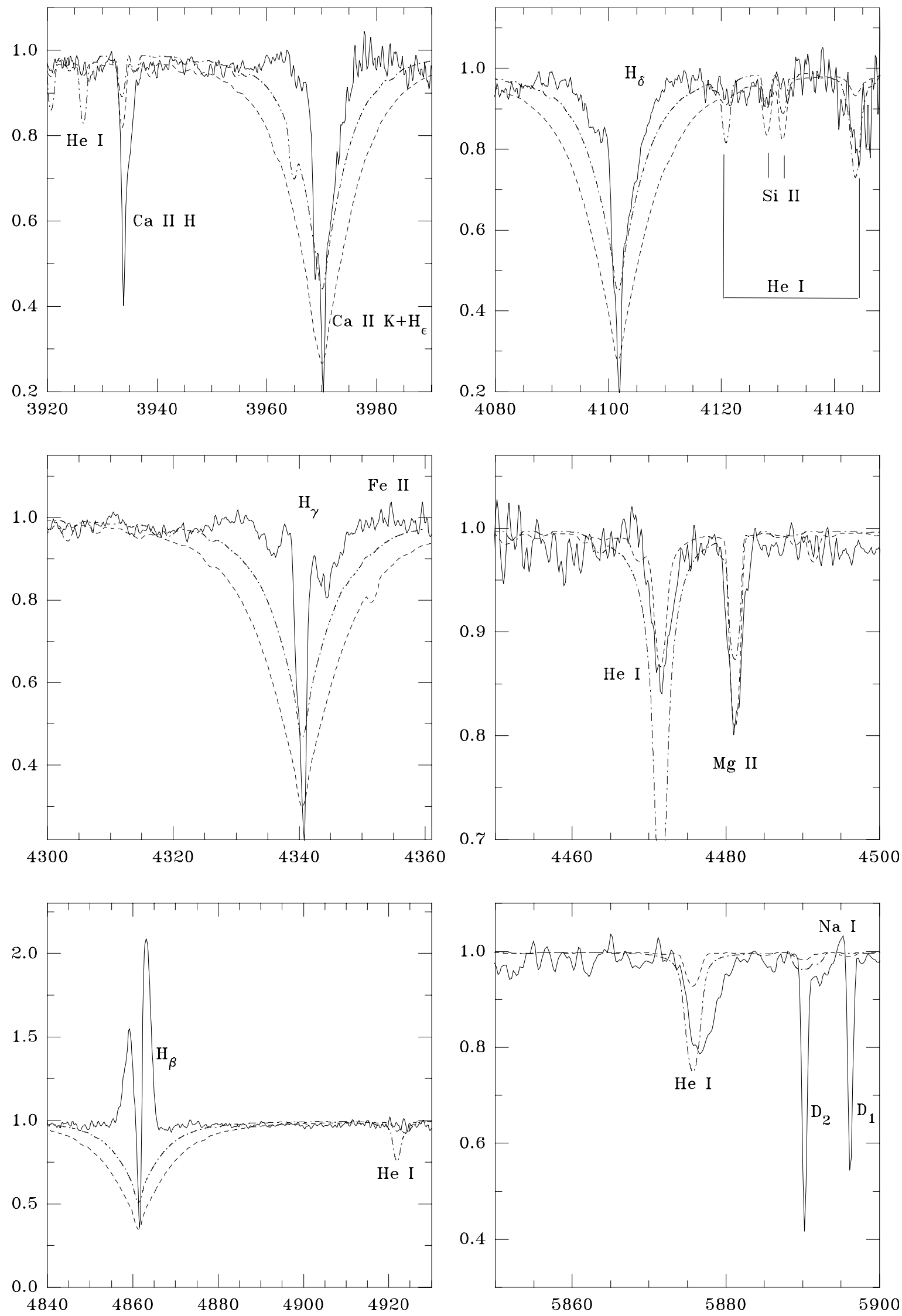

Fig. 3. Parts of the spectrum of Hen 3-140. The star's spectrum is shown by solid lines, while the theoretical ones is shown by dashed lines (for $T_{\text {eff }}=12000 \mathrm{~K}, \log g=4.0$, and $v \sin i=70 \mathrm{~km} \mathrm{~s}^{-1}$ ) and by dash-dotted line (for $T_{\text {eff }}=19000 \mathrm{~K}, \log g=3.5$, and $\left.v \sin i=70 \mathrm{~km} \mathrm{~s}^{-1}\right)$. The intensity and wavelength units are the same as in Fig. 2 


\section{Analysis of the observational data}

\subsection{Spectra}

The lines in the objects' spectra were identified using the catalogue by Coluzzi (1993). Since the noise at the échelle order edges $(\sim 20 \AA$ from each edge) is higher than in the remaining areas and the overlapping regions are small $(\sim 30 \AA)$, some spectral lines might have been missed.

\subsubsection{HD 85567 and Hen 3-140}

Spectra of HD 85567 and Hen 3-140 are similar and contain Balmer lines in emission, a small number of weak emission Fe II lines, He I lines in absorption, and a few other absorption lines characteristic of B-type stars. Weak diffuse interstellar bands (DIBs) at 5780 and $5797 \AA$ are seen in the spectra of both objects. Parts of the blue region spectra of HD 85567 and Hen 3-140 are shown in Figs. 2 and 3, respectively, while the characteristics of the identified lines are listed in Table 3.

Since both stars show a mild reddening, one can estimate their spectral type from the photometric data. The optical colour-indices corrected for the interstellar reddening using the standard extinction law (Savage \& Mathis 1979) correspond to B2 V spectral type for HD 85567 and B7 $\mathrm{V}$ for Hen 3-140. In order to check these estimates, we calculated theoretical spectra in the regions of the observed lines using the radiation transfer code SYNSPEC (Hubeny et al. 1995) for the stellar parameters listed in Table 4 . The values of $v \sin i$ are estimated by comparison of the absorption (HD 85567 and Hen 3-140) or emission (Hen 3-1398) line widths with those in the theoretical spectra (see Figs. 2, 3, and 5).

The spectrum of HD 85567, obtained in the red region, contains many emission features including a strong $\mathrm{H} \alpha$ line (see Fig. 4 and Table 5) and a number of Pashen lines (P12-P22), the 7771/5 triplet of neutral oxygen, forbidden oxygen lines at 6300 and $6364 \AA$, and a strong IR triplet of Ca II. There are a few weak absorption lines (Si II 6371 and Ne I $6402 \AA$ ) which are marginally detected because of a rather low signal-to-noise ratio. The line characteristics in the region overlapping with the blue spectrum (5750-5900 $\AA$ ) show no signs of variations between the two observations.

The comparison of the observed and theoretical line profiles shows that only a few absorption lines (Mg II 4481, Si II $4128 \& 4130 \AA$, and He I 4026 and $4471 \AA$ ) can be satisfactorily fitted by the models. However, comparison of all fitted lines or their wings (in case of the Balmer lines) gives controversial results. For example, for HD 85567 the intensity ratio of the He I lines at 4009 and $4026 \AA$ and that of the He I 4471 to Mg II $4481 \AA$ suggest the B7 spectral type, while the Balmer line wings favor the B2 type (see Fig. 2). This situation is reversed in Hen $3-140$. The $\mathrm{H} \beta$ line in its spectrum shows no traces of the photospheric wings at all. However, if we assume that they are seen in $\mathrm{H} \gamma$ and $\mathrm{H} \delta$, an effective temperature ( $\left.T_{\text {eff }}\right)$ of at least
$20000 \mathrm{~K}$ is required to explain them. At the same time, the theoretical He I $4471 / \mathrm{Mg}$ II 4481 ratio is close to the observed one for the B7 type, but the observed He I $5876 \AA$ and $\mathrm{Ca}$ II $\mathrm{H}$ lines are much weaker than the theoretical predictions for this spectral type.

These discrepancies are most likely due to the effect of circumstellar matter on the observed spectrum, which causes both a distortion and variability of the line profiles. The latter was found to be the case for HD 45677, a Be star with warm dust (Israelian et al. 1996). Thus we accept the spectral types following from the photometry in the case of Hen 3-140 and from the photometry and the Balmer line wings fitting for HD 85567.

\subsubsection{Hen 3-1398}

The spectrum of Hen 3-1398 is significantly different from those of the other 2 objects, except for the appearance of the Fe II emission lines. All detected He I lines are in emission and have narrow $\left(F W H M \sim 30-60 \mathrm{~km} \mathrm{~s}^{-1}\right)$ singlepeaked profiles. The strongest members of the Balmer series $(\mathrm{H} \beta$ and $\mathrm{H} \gamma)$ display triple-peaked emission profiles. The other Balmer lines in the observed region are filled-in with emission, and it is more difficult to constrain their profiles because of a lower signal-to-noise ratio and contamination by other lines. A strong He II $4686 \AA$ line, which points to a high temperature of the star, and weak [O III] 4959 and $5007 \AA$ lines are also present. The extreme weakness of the Si II lines at 4128 and $4130 \AA$ and the strength of the $\mathrm{Mg}$ II $4481 \AA$ line suggest that the star's spectral type is not later than B0. The characteristics of the identified lines in the spectrum of Hen 3-1398 are listed in Table 6 .

Parts of the spectrum of Hen 3-1398 and corresponding theroretical spectra calculated with the SYNSPEC code for the star's parameters from Table 4, are shown in Fig. 5. The adopted $T_{\text {eff }}$ and gravity are not the best fit parameters as our spectrum has a moderate signal-to-noise ratio and only a few absorption line were detected. Nevertheless, the spectral features discussed above suggest that $T_{\text {eff }}$ is not lower than $\sim 30000 \mathrm{~K}$. The Balmer line wings, which are barely seen in the $\mathrm{H} \gamma$ and $\mathrm{H} \delta$ profiles, are consistent with such a high temperature. As in the case of HD 85567 and Hen 3-140, the spectrum itself does not allow us to judge the luminosity of Hen 3-1398. However, as we will show in Sect. 5, the object is close to the ZAMS and $\log g \sim 4.0$ is a reasonable estimate for its gravity.

\subsection{Spectral energy distributions}

The SED morphology of all three objects is the same as for other Be stars with warm dust. In the UV and optical region it is controlled by the stellar radiation; a noticeable peak is seen in the near-IR which is followed by a rapid flux decrease longward of $\sim 10 \mu \mathrm{m}$. The SEDs of all the objects constructed from the available photometric data are shown in Fig. 6. In order to construct the SED of 

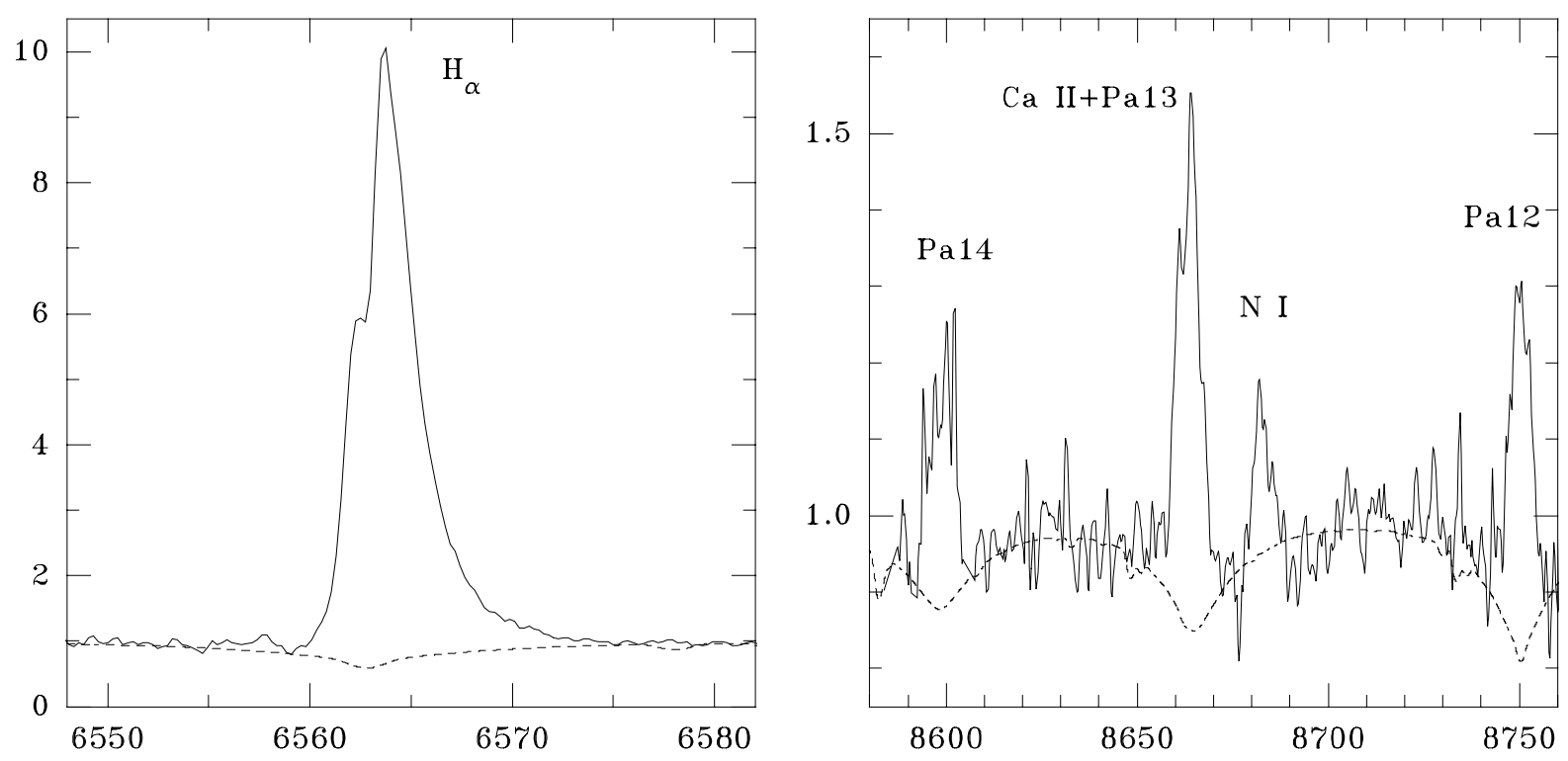

Fig. 4. Portions of the red spectrum of HD 85567. The stellar spectrum is shown by solid lines, while the theoretical one (for $T_{\text {eff }}=19000 \mathrm{~K}, \log g=3.5$, and $v \sin i=50 \mathrm{~km} \mathrm{~s}^{-1}$ ) is shown by dashed lines. he intensity and wavelength units are the same as in Fig. 2

Table 5. Lines identified in the red spectrum of HD 85567

\begin{tabular}{llllll}
\hline Line & $\lambda_{\text {lab }}$ & $I / I_{\mathrm{c}}$ & $R V$ & $E W$ & Rem. \\
\hline DIB & 6278. & $0.90:$ & & 0.41 & \\
DIB & 6283.85 & 0.92 & -1 & 0.08 & \\
[O I](1) & 6300.23 & 2.73 & -5 & 1.27 & single emis. \\
[O I](1) & 6363.88 & $1.60:$ & -13 & 0.53 & single emis. \\
Si II(2) & 6371.36 & $0.90:$ & -4 & 0.06 & single abs. \\
Ne I (1) & 6402.25 & $0.93:$ & -13 & 0.06 & single abs. \\
H $\alpha$ & 6562.82 & 10.1 & & 30.7 & a \\
O I (1) & 7771.96 & 1.12 & -34 & & b \\
O I (1) & 7774.18 & 1.26 & -31 & & b \\
Pa 22 & 8359.01 & $1.10:$ & & & \\
Pa 21 & 8374.48 & $1.12:$ & & & \\
Pa 20 & 8392.40 & 1.18 & & & \\
Pa 19 & 8413.32 & $1.34:$ & & & \\
Pa 18 & 8437.96 & $1.25:$ & & & \\
O I (4) & 8446.76 & $1.60:$ & & & \\
Pa 14 & 8598.39 & $1.1:$ & & 2.6 & \\
Ca II(2)+Pa 13 & 8662.14 & 1.6 & $+37:$ & 4.1 & c \\
N I (1) & 8680.24 & 1.2 & & & b \\
Pa 12 & 8750.48 & $1.1:$ & -13 & 1.8 & \\
\hline
\end{tabular}

a - The equivalent width is related to the emission component only.

$\mathrm{b}$ - All three lines in the triplet are blended.

c - Other lines of the Ca IR triplet were not detected due to the gaps between the échelle orders.

Hen 3-140, we used unpublished $U B V(R I)_{\mathrm{c}}$ data, which were kindly provided by Dolf de Winter (priv. communication). It is seen in the middle panel of Fig. 6 that our near-IR data obtained for Hen 3-140 for the first time revealed a strong near-IR excess.

Corrections for reddening were applied to the data using the results of the spectral analysis. The overall
Table 6. Lines identified in the spectrum of Hen 3-1398

\begin{tabular}{llllll}
\hline Line & $\lambda_{\text {lab }}$ & $I / I_{\mathrm{c}}$ & $R V$ & $E W$ & Rem. \\
\hline He I (20) & 3867.63 & 2.35 & +73 & 0.81 & single emis. \\
He I (2) & 3888.65 & $1.2:$ & -11 & 0.13 & a \\
Ca II H (1) & 3933.66 & 0.41 & -20 & 0.32 & single abs. \\
He II (3) & 3968.43 & $1.5:$ & $-88:$ & $0.8:$ & b \\
He I (18) & 4026.18 & 1.17 & -15 & 0.30 & single emis. \\
O II(48) & 4089.30 & 0.65 & -82 & 0.06 & single abs. \\
H $\delta$ & 4101.74 & & & & comp. \\
He I (16) & 4120.81 & 1.30 & -5 & 0.06 & single emis. \\
H $\gamma$ & 4340.47 & 1.27 & & 1.07 & comp. \\
He I (14) & 4471.48 & 1.54 & -12 & 0.37 & single emis. \\
Mg II (4) & 4481.13 & $0.92:$ & $+13:$ & 0.08 & single abs. \\
Fe II (37) & 4555.89 & 1.10 & & 0.15 & dp: \\
Fe II (37) & 4582.84 & 1.10 & & 0.59 & dp \\
He II (1) & 4685.68 & 3.33 & -16 & 2.49 & single emis. \\
He I (12) & 4713.14 & 1.13 & -9 & 0.14 & single emis. \\
H $\beta$ He I (48) & 4861.33 & 1.89 & & 4.37 & comp. \\
Fe II (42) & 4921.93 & 1.20 & -3 & 0.22 & c \\
[O III](1) & 4958.92 & 1.06 & & 0.15 & comp.: \\
[O III](1) & 5006.84 & 1.06 & -1 & 0.05 & single emis. \\
He II (4) & 5015.68 & 1.45 & -13 & 0.13 & single emis. \\
Fe II (42) & 5018.43 & 1.12 & -13 & 0.43 & single emis. \\
Si II (5) & 5041.06 & 1.07 & -28 & 0.10 & d single emis. \\
Fe II (49) & 5234.62 & 1.08 & -3 & 0.21 & dp \\
Fe II (49) & 5316.61 & 1.14 & -6 & 0.40 & e \\
Fe II (48) & 5362.86 & 1.06 & -5 & 0.20 & dp \\
\hline
\end{tabular}

a - Inside the $\mathrm{H} 8$ absorption profile.

b - Blend with the Ca II $\mathrm{K}+\mathrm{H} \epsilon$ absorptions.

c - Blend with the Fe II (42) $4923 \AA$.

d - Double-peaked and slightly in blend with the He I $5016 \AA \AA$.

e - Double-peaked, a contribution from the Fe II (48) $5316.78 \AA$ is possible. 

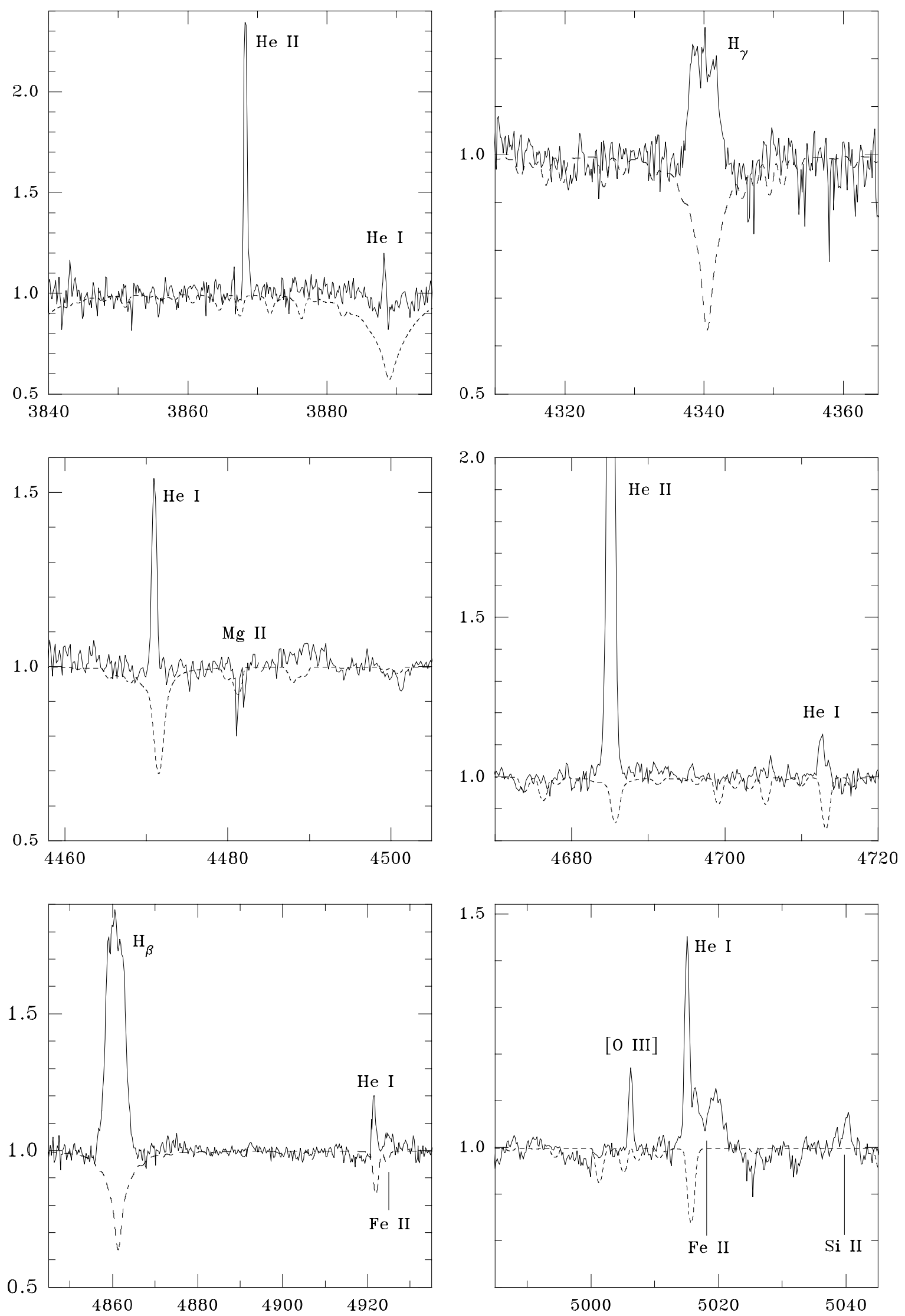

Fig. 5. Parts of the spectrum of Hen 3-1398. The stellar spectrum is shown by solid lines, while the theoretical one (for $T_{\text {eff }}=30000 \mathrm{~K}, \log g=4.0$, and $v \sin i=50 \mathrm{~km} \mathrm{~s}^{-1}$ ) is shown by dashed lines. The intensity and wavelength units are the same as in Fig. 2 
interstellar and circumstellar extinction was taken into account assuming the same wavelength dependence for both components. This assumption is reasonable given a comparatively small amount of the circumstellar contribution (see Sect. 4.1). The de-reddened SEDs for all three objects are in good agreement with the theoretical spectra for the corresponding spectral types, derived above.

As mentioned in Sect. 3.2, the IR excess of HD 85567 was fitted with a two-component optically-thin dusty envelope model by Malfait et al. (1998). However, such a SED can also be fitted by an optically-thick and geometrically-thin gaseous-and-dusty disk (Bertout et al. 1988) with an outer temperature $\sim 100-200 \mathrm{~K}$ (see Miroshnichenko \& Corporon 1999). The same is true for Hen 3-140 and Hen 3-1398. The appearance of a weak $10-\mu \mathrm{m}$ silicate emission feature in the spectrum of Hen 3-1398 indicates that a certain amount of optically thin dust is present in the circumstellar envelope. Thus, to explain the overall SED, two-component envelope models, which combine optically-thin and optically-thick parts, can be applied. Examples include an optically-thick disk + optically-thin spherical envelope model by Miroshnichenko et al. (1999), and an opticallythick accretion disk with an optically-thin surface layer by Chiang et al. (2001). However, the main problem hampering conclusive modelling is that the IR data obtained so far for all three objects are not sufficient to choose between different possible models. For example, the disk, which is cooler than the envelope, usually controls the SED at $\lambda \geq 100 \mu \mathrm{m}$ where we have no data available. Furthermore, the near-IR peak of the SED may be explained either by an optically-thin dust emission of any geometry or with an optically-thick disk emission. Finally, only imaging at IR wavelengths is capable of discriminating between an extended, optically-thin, mostly spherical component and a more compact disk. Therefore, we are not able to conclude which dusty configuration is more appropriate to explain the IR excesses of the objects in question. Future far-IR and millimeter wavelength photometry, IR spectroscopy, and imaging are needed to resolve this problem. In any case, the steep decrease of the far-IR flux with wavelength implies that the dusty envelopes are compact in all three objects.

\section{Discussion}

The observational results derived in the previous section show that we are dealing with peculiar objects, which require separate discussions about derivation of their physical properties.

\subsection{Fundamental parameters}

\subsubsection{HD 85567}

The information about HD 85567 analysed above indicates that its most likely spectral type is B2. The $A_{V}$ derived from the DIBs strength is significantly smaller than that derived from $U-B$ and $B-V(0.5$ and $1.2 \mathrm{mag}$, respectively). The latter fact suggests the presence of an additional, circumstellar, reddening.

Since no luminosity indicators were found in the object's spectrum, we will use different indirect methods to estimate the distance towards HD 85567 and, hence, its luminosity. The average radial velocity $(R V)$ of the absorption lines (He I, Ca II, Mg II) in the spectrum of HD 85567 is $0 \pm 2 \mathrm{~km} \mathrm{~s}^{-1}$. This value lies between the mean $R V$ of the stars closest to the Sun within $2^{\circ}$ of the object $\left(\sim+20 \mathrm{kms}^{-1}\right.$ at $\left.D=100-200 \mathrm{pc}\right)$ and the mean $R V$ of the association Car OB1, which is located within $5^{\circ}$ of the object $\left(-5 \mathrm{~km} \mathrm{~s}^{-1}\right.$ at $\sim 2.5 \mathrm{kpc}$ from the Sun, Humphreys 1978). The galactic rotation curve (Dubath et al. 1988) predicts a decrease of $R V$ from +20 to $+8 \mathrm{~km} \mathrm{~s}^{-1}$ with $D$ in the range from 0.1 to $2 \mathrm{kpc}$ from the Sun, but it does not reproduce the observed negative $R V$ beyond this region and cannot be used as a distance estimator in this case. A lower limit for the distance toward HD 85567, $1 \mathrm{kpc}$, is set by the ZAMS luminosity for the adopted $T_{\text {eff }}$. An upper limit of $\sim 2 \mathrm{kpc}$ is constrained by the location of the front edge of the Carina arm (e.g., Grabelsky et al. 1988), inside which hot stars are generally more reddened than HD 85567 (Humphreys 1978). Thus, the star is most probably located at $D=$ $1.5 \pm 0.5 \mathrm{kpc}$. This is in agreement with the interstellar extinction law in its direction $\left(A_{V}=1.0 \pm 0.5\right.$ at $D \geq 1 \mathrm{kpc}$, Neckel \& Klare 1980) and implies a bolometric luminosity $\log L_{\mathrm{bol}} / L_{\odot}=3.7 \pm 0.2$.

\subsubsection{Hen $3-140$}

The spectral type of Hen 3-140 is somewhat uncertain. The $U B V$ photometry, which roughly measures the Balmer jump, suggests B7, while the apparently narrow wings of the Balmer lines favor an earlier type, about B2. Adoption of the later type gives the overall reddening toward the object in agreement with the interstellar $E_{B-V}$ estimate from the DIB strengths at $\lambda \lambda 5780$ and $5797 \AA$ ( $\left.A_{V}=0.95 \pm 0.05 \mathrm{mag}\right)$. If we adopt the earlier type, we would then need to account for the lack of the UV radiation. A reasonable explanation is that the circumstellar envelope is optically thick in the Balmer continuum. This might imply that all the optical colour-indices are affected by the circumstellar contribution and make the photometric reddening estimate unreliable. On the other hand, in similar objects even with stronger emission lines (e.g., HD 45677), this does not seem to be the case. Thus, we will not attempt to resolve this problem until more comprehensive data including direct Balmer jump measurements are obtained.

The luminosity of Hen 3-140 can be derived through indirect distance estimates, as in case of HD 85567. The average $R V$ of Hen 3-140 based on the He I, Ca II, and Fe II lines, is $+33 \pm 2 \mathrm{kms}^{-1}$. This leads to a distance $D=2.8 \pm 0.2 \mathrm{kpc}$ toward the star using the galactic rotation curve (Dubath et al. 1988). This method 
predicts a $R V$ increase with distance from the Sun in this direction. Indeed, the mean $R V$ of the closest OB association to Hen 3-140 in the sky, Vela OB1, which is located at $1.8 \mathrm{kpc}$ from the Sun, is $+25 \mathrm{~km} \mathrm{~s}^{-1}$. However, Vela OB1 is much closer to the galactic plane $(-2.7 \leq b \leq+1.4)$, and the galactic rotation constants are determined using stars within the distance $z= \pm 350 \mathrm{pc}$ from it. Therefore, the applicability of this method may be limited as the object is located relatively far from the galactic plane $(b=$ -8.5). The interstellar extinction shows a linear increase in the object's direction and reaches $E_{B-V} \sim 0.2 \mathrm{mag}$ at $D=1 \mathrm{kpc}$ (Eggen 1986). Thus, a first order distance estimate toward Hen $3-140$ is $D=2.0 \pm 0.5 \mathrm{kpc}$, which corresponds to the luminosity $\log L_{\mathrm{bol}} / L_{\odot}=3.1 \pm 0.2$. This is comparable to the luminosities of non-supergiant Be stars with warm dust regardless of the adopted object's $T_{\text {eff }}$ (Sheikina et al. 2000; see Fig. 8).

\subsubsection{Hen 3-1398}

The spectrum of Hen 3-1398 permits a better estimate of its spectral type than those of the other 2 objects. With good confidence we adopt it here as O9/B0. Again, as above, we will get the object's luminosity through the distance estimate. Hen $3-1398$ is very close to the galactic plane $(b=-1.7)$, therefore the galactic rotation curve method is expected to work in its case. The method seems to be reliable unless the object has a peculiar velocity comparable to or higher than the velocity due to the galactic rotation. The mean $R V$ of 7 He I single-peaked narrow emissions is $-10 \pm 3 \mathrm{~km} \mathrm{~s}^{-1}$, which corresponds to $D=3.3 \pm 0.4 \mathrm{kpc}$. The interstellar extinction law we derived using photometry and spectral types of stars with $1^{\circ}$ from the position of Hen 3-1398 (Fig. 7) shows an approximately linear increase of $E_{B-V}$ at $D \leq 2 \mathrm{kpc}$. Beyond this distance no additional reddening is seen, apparently due to passing the outer edge of the Scorpius arm. This point suggests a lower limit of $D$ for the object. Using the derived kinematical estimate for $D$, the resulting object's luminosity is $\log L_{\mathrm{bol}} / L_{\odot}=5.3 \pm 0.2$. Thus, Hen 3-1398 is located within the main sequence being the hottest and the most luminous among non-supergiant Be stars with warm dust (see Fig. 8).

\subsection{Circumstellar envelopes}

The emission-line profiles of all three stars suggest that they are formed in non-spherical circumstellar envelopes. The Balmer lines of HD 85567 show a double-peaked structure with a moderate central depression and an additional strong absorption component at $R V=-264 \pm$ $12 \mathrm{kms}^{-1}$ (average from $\mathrm{H} \beta, \mathrm{H} \gamma, \mathrm{H} \delta$, and $\mathrm{H} \epsilon$ ), which is rather unusual in this profile type. The $\mathrm{H} \alpha$ line profile in our red spectrum (see Fig. 4) has the same shape but with a much weaker additional absorption at $R V=$ $-180 \mathrm{~km} \mathrm{~s}^{-1}$. The emission components of the Balmer and Fe II lines are significantly redshifted with respect to the

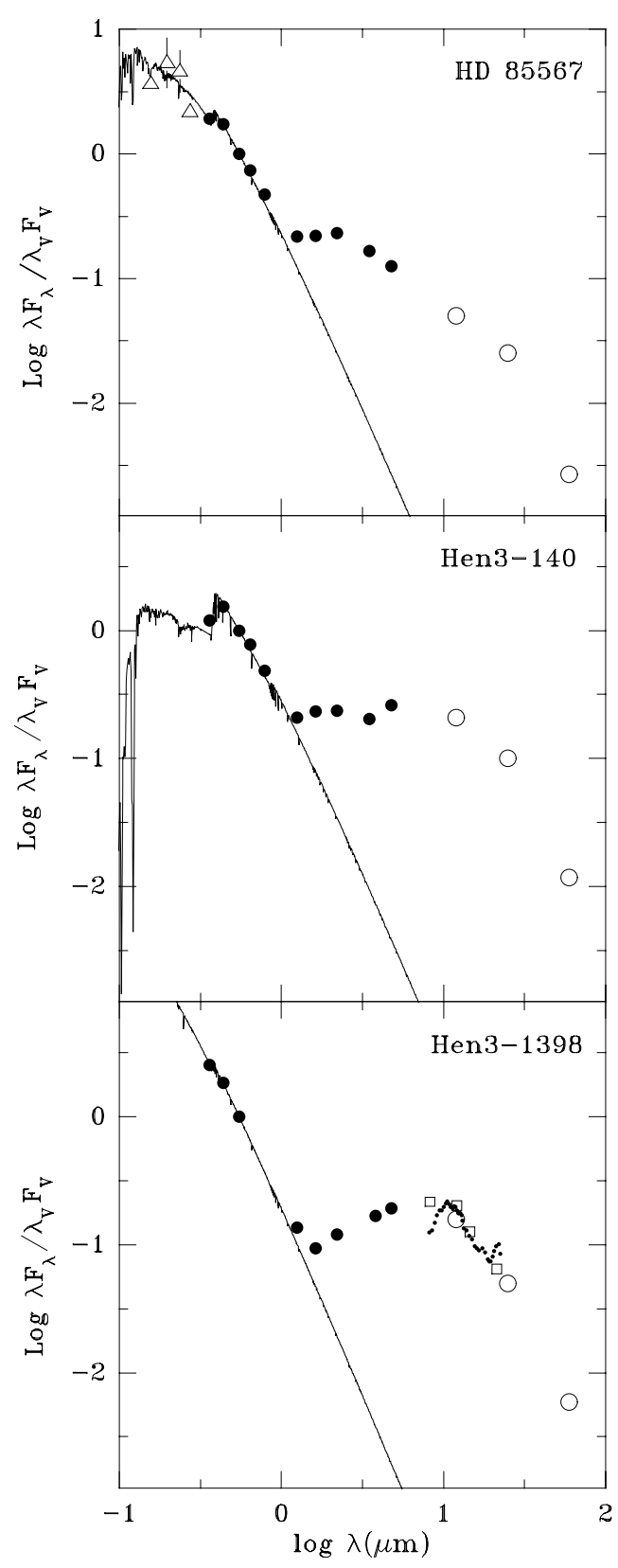

Fig. 6. Spectral energy distributions. The reddening correction was applied using the extinction values derived from the photometry and adopted spectral types. The IRAS filter photometry is shown by open circles, the ground-based data by filled circles. The TD-1 UV data for HD 85567 are shown by open triangles, the MSX data for Hen 3-1398 by open squares, while the IRAS LRS spectrum of Hen 3-1398 is shown by dots. Unless explicitly shown, the flux uncertainties are of the order of the symbol size. Solid lines represent stellar intrinsic SEDs from Kurucz (1994) for the fundamental parameters from Table 4

absorption lines $\left(\Delta R V \geq 40 \mathrm{~km} \mathrm{~s}^{-1}\right)$. All these facts indicate a complex structure of the envelope.

The Balmer lines of Hen 3-140 have double-peaked profiles with a strong central depression, which might imply an almost edge-on orientation of the envelope. The Fe II lines are certainly present but are very weak. 


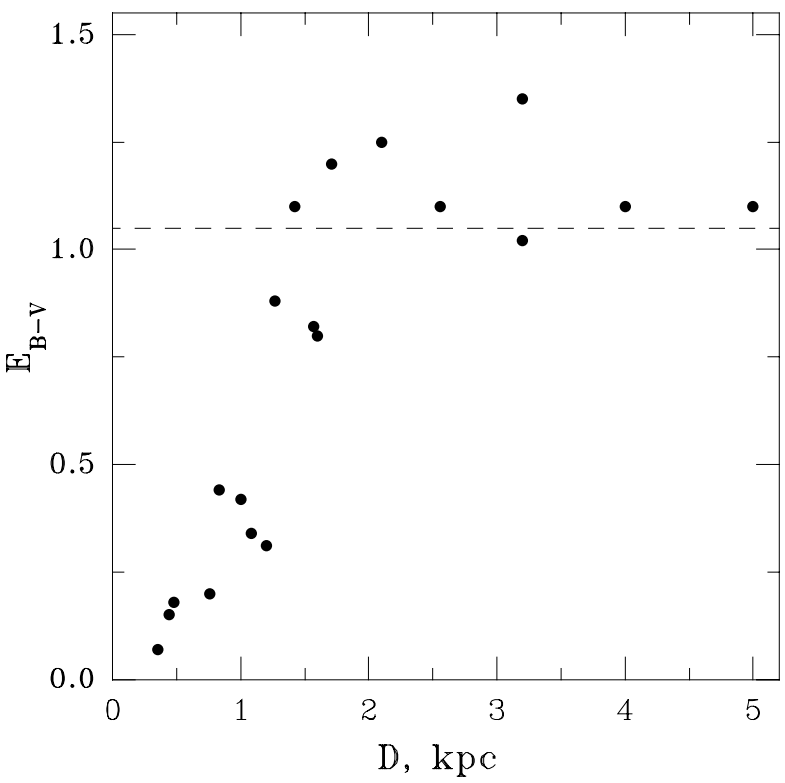

Fig. 7. The interstellar extinction law in the vicinity of Hen 3 1398. The dashed line represents the colour-excess of the object

The envelope's asphericity is also suggested by the presence of the intrinsic polarization (Yudin \& Evans 1998).

As we described above, the emission line profiles of different species in the spectrum of Hen 3-1398 are different. The He I and He II lines, which are formed very close to the star, are narrow and single-peaked. They may originate in an optically-thin spherical-like envelope or in a faceon disk. The Fe II lines are formed further away and are double-peaked with the peak separation about $185 \mathrm{~km} \mathrm{~s}^{-1}$, that is roughly the same as the blue-red peak separation in the Balmer lines. The shallow absorption components between the emission peaks in the Fe II lines suggest the presence of a disk inclined at an intermediate angle with respect to the line of sight. The Balmer line central peaks have the same $R V$ as the He lines and may originate in the same envelope regions. One of the explanations for such behaviour may be that the double-peaked structure and the narrow single-peaked one are formed in the envelopes of different components of a binary system. Such a scenario has been suggested by Křiž \& Harmanec (1985) for binary Be stars where one component is surrounded by a disk, while another one by a mostly spherical cloud of gas. Triple-peak Balmer line profiles are indeed observed in the spectra of some Be binaries, such as $\zeta$ Tau (e.g., Hanuschik et al. 1996).

The IR excess shape in the objects' SEDs was already discussed in Sect. 4.2. It is consistent with a non-spherical distribution of the circumstellar dust, which needs to be optically-thick to account for the large IR-fluxes. This suggestion is supported by the shape of the spectral line profiles. However, the presence of an additional, opticallythin and more extended, dusty component cannot be excluded, as the $10-\mu \mathrm{m}$ silicate emission feature is seen in the spectrum of Hen 3-1398. New mid-IR spectroscopic observations of all the objects and detailed modelling are needed to draw a more certain conclusion about the dust geometry.

\subsection{Nature and evolutionary state}

From the above analysis we conclude that all three objects are most likely located within the main-sequence part of the HRD. In general, this implies several possibilities for their evolutionary state. They might be pre-mainsequence Herbig Be stars, as was suggested for HD 85567 by Malfait et al. (1998). However, the lack of a far-IR excess in comparison with most Herbig $\mathrm{Ae} / \mathrm{Be}$ stars does not support this suggestion. Moreover, the fact that these authors described the IR excess of HD 85567 as doublepeaked is not consistent with their own evolutionary scenario, which implies that the star should be very close to the ZAMS. Even cooler Herbig Ae/Be stars retain significant far-IR excesses almost at ZAMS (e.g., van den Ancker et al. 1998). At the same time, Herbig Be stars with masses comparable to that of HD 85567 and rather short evolutionary times at the pre-main-sequence stage (e.g., HD 200775) also display very strong far-IR excesses. We should note here that only a few objects belong to this group; most of them have been proven to be binaries (e.g., Millan-Gabet et al. 2001), although whether they are really pre-main-sequence objects is not clear yet. Potentially, HD 85567 can belong to this group.

The objects might be post-main-sequence stars on the horizontal post-AGB track. However as discussed in Miroshnichenko et al. (2000), such objects are known to be strong emitters in the far-IR, with peak emission between 25 and $60 \mu \mathrm{m}$. They are located in a very different region of the IRAS colour-colour diagramme (e.g., GarcíaLario et al. 1997).

Emission-line spectra and IR-excesses similar to those of our objects are observed in $\mathrm{B}[\mathrm{e}]$ supergiants (e.g., Zickgraf et al. 1986). In the optical region the latter show narrow low-excitation emission lines, while high-excitation features are seen in the UV region. Such behaviour suggests a two-component model for the stellar wind which includes a slow, dense and cool equatorial wind and a fast, hot and rapid polar wind (Zickgraf et al. 1986). Among our objects, only HD 85567 was observed by the IUE satellite in its long-wavelength region in the low-resolution mode ( $\lambda \lambda$ 1850-3350 A, LWP19729 and LWP20659). Neither IUE spectrum shows prominent high-excitation features, although they were obtained with a rather low signal-tonoise ratio and a large portion of the spectra $(\lambda \lambda 2300-$ $3100 \AA$ ) is unusable.

Another problem with this interpretation is that our objects most likely have much lower luminosities than $\mathrm{B}[\mathrm{e}]$ supergiants, unless we significantly underestimated their distances. This might be the case for Hen 3-1398, but not for HD 85567 or Hen 3-140. A large luminosity increase required would put HD 85567 into the Carina arm, where interstellar extinction is noticeably larger than observed. Even a larger distance ( $\sim 6 \mathrm{kpc})$ is needed for Hen 3-140 to 


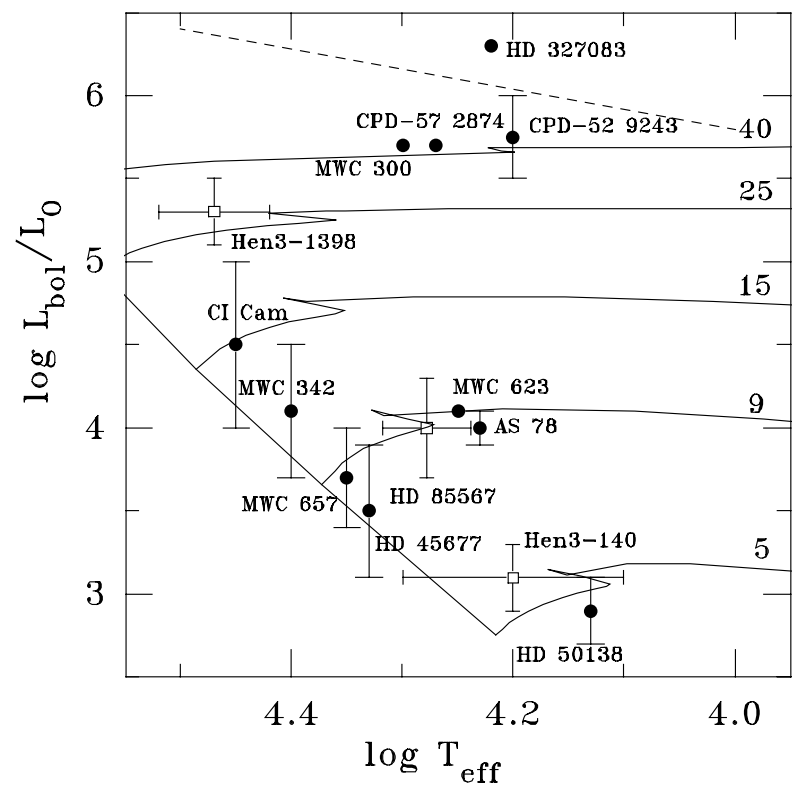

Fig. 8. The Hertzsprung-Russell diagram. Evolutionary tracks from Schaerer et al. (1993) are shown by solid lines. The ZAMS connects the starting points of all the tracks used. Initial masses in solar units are indicated near corresponding tracks. The Humphreys-Davidson limit is shown by a dashed line. Filled circles represent positions of Be stars with warm dust

be a supergiant. Its galactic latitude would be translated into a distance of about $1 \mathrm{kpc}$ from the galactic plane, which is not consistent with the concept of a disk population star, such as most massive stars.

Thus, the most likely possibility is that all three objects are main-sequence stars. Their emission-line spectra are similar to those of classical Be stars, although the latter have mostly free-free IR excesses. Only a few classical Be stars have been suspected of having distant and cold circumstellar dust in their environments (Miroshnichenko \& Bjorkman 2000), but this only results in existence of a far-IR excess. Another difference of our objects from classical Be stars is much lower rotational velocities (see Table 4). The observed double-peaked Balmer line profiles suggest that the orientation of the circumstellar envelopes is far from pole-on, and the derived values of $v \sin i$ should not be much lower that the total rotational velocity. It seems to be difficult to produce a noticeable amount of circumstellar matter through matter ejection from a single star at such a slow rotation rate (cf. Bjorkman 2000 for a recent review).

Summarizing the discussions about the objects' properties, and taking into account that some Be stars with warm dust are known to be binaries (e.g., CI Cam, MWC $623)$, we might suggest that the objects of this study may be main-sequence binaries, too. However, this is just a working hypothesis as no strong evidence for such a conjective has been found so far. In order to detect traces of the orbital motion in such systems, a long-term monitoring is required.

\section{Conclusions}

New optical spectroscopic and near-IR photometric data are obtained and analysed for three early-type stars (HD 85567, Hen 3-140, and Hen 3-1398) which have a large excess radiation in the IRAS spectral region. Our new near-IR observations confirmed the existence of a large excess in HD 85567 earlier detected by Malfait et al. (1998). They also resulted in the detection of an even larger excess in Hen 3-140.

All three objects of this study are found to have peculiar spectral line profiles, which are apparently formed in non-spherical envelopes. The presence of circumstellar polarization in Hen 3-140 also favors the envelope nonsphericity. The shape of the IR excesses indicates that the circumstellar dust distribution is rather compact, but a conclusion about its geometry is premature.

The derived interstellar reddening, distances, and luminosities of the objects suggest that they are intermediate-mass stars which are located within the main-sequence. The objects are neither classical Be stars (because of the large near-IR excesses) nor pre-mainsequence Herbig Be stars (because of their location far from the ZAMS; lack of signatures of cold dust, nebulosities, and close star forming regions). Also they are unlikely to be $\mathrm{B}[\mathrm{e}]$-supergiants or post-AGB stars. The whole set of their properties resemble that of a recently defined group of Be stars with warm dust (Sheikina et al. 2000).

The complex structure of the Balmer line profiles and a large difference between the $R V$ of the absorption and emission lines in HD 85567 and Hen 3-1398 might imply that they have binary components. Testing such a hypothesis requires additional high-resolution spectroscopic observations in order to search for the $R V$ variations. Photometry, spectroscopy, and imaging in the IR and millimetre-wavelength regions are important to constrain the circumstellar envelope properties. Spectroscopy in the UV region may be helpful in constraining properties of the central stars.

Acknowledgements. This paper uses observations made at the South-African Astronomical Observatory (SAAO). We thank T. Lloyd Evans, D. Kilkenny, F. Marang, and F. Van Wyk for obtaining the multicolour photometry of HD 85567 and Hen 3-140 at SAAO as well as D. de Winter for sending us his unpublished photometric data. A. M. and K. S. B. acknowledge support from NASA grant NAG5-8054 and thank the IRTF staff for their assistance during the observations. Karen Bjorkman is a Cottrell Scholar of the Research Corporation, and gratefully acknowledges their support. This research was partially supported by a grant to H. L. from FONCYT (Fondo Nacional para la Ciencia y Tecnologia), PICT 1505.

\section{References}

Allen, D. A., \& Swings, J.-P. 1976, A\&A, 47, 293

van den Ancker, M. E., de Winter, D., \& Tjin A Djie, H. R. E. 1998, A\&A, 330, 145

Bertout, C., Basri, G., \& Bouvier, J. 1988, ApJ, 330, 350 
Bjorkman, J. E. 2000, in The Be-phenomenon in Early-Type Stars, ed. M. A. Smith, H. F. Hendrichs, \& J. Fabregat, ASP Conf. Ser., 214, 435

Chiang, E. I., Joung, M. K., Greech-Eakman, M. J., et al. 2001, ApJ, 547, 1077

Coluzzi, R. 1993, Bull. Inf. CDS, 43, 7

Drilling, J. S. 1991, ApJS, 76, 1033

Dubath, P., Mayor, M., \& Burki, G. 1988, A\&A, 205, 77

Egan, M. P., et al. 1999, The Midcourse Space Experiment Point Source Catalog Version 1.2, Explanatory Guide, AFRL-VS-TR-1999-1522

Eggen, O. J. 1986, AJ, 92, 1074

ESA 1997, The Hipparcos and Tycho Catalogues ESA SP-1200

García-Lario, P., Manchado, A., Pych, W., \& Pottasch, S. R. 1997, A\&AS, 126, 479

Garrison, R. F., Hiltner, W. A., \& Schild, R. E. 1977, ApJS, 35,111

Grabelsky, D. A., Cohen, R. S., Bronfman, L., \& Taddeus, P. 1988, ApJ, 331, 181

Guglielmo, F., Epchtein, N., Le Berte, T., et al. 1993, A\&AS, 99,31

Hanuschik, R. W., Hummel, D., Sitorius, E., Dietle, O., \& Thimm, G. 1996, A\&AS, 116, 309

Henize, K. G. 1976, ApJS, 30, 491

Houk, N., \& Cowley, A. P. 1975, Michigan Spectral Survey, Ann Arbor, Dep. Astron., Univ. Michigan, 1

Hubeny, I., Lanz, T., \& Jeffery, C. S. 1995, Synspec - A User's Guide

Humphreys, R. M. 1978, ApJS, 38, 309

Israelian, G., Friedjung, M., Graham, J., et al. 1996, A\&A, 311,643

Klare, G., \& Neckel, Th. 1977, A\&AS, 27, 215

Kozok, J. R. 1985a, A\&AS, 61, 387

Kozok, J. R. 1985b, A\&AS, 62, 7
Křiž, S., \& Harmanec, P. 1975, Bull. Astron. Inst. Czech., 26, 65

Lada, C., \& Adams, F. 1992, ApJ, 393, 278

Lamers, H. J. G. L. M., Zickgraf, F.-J., de Winter, D., Houziaux, L., \& Zorec, J. 1998, A\&A, 340, 117

Malfait, K., Bogaert, E., \& Waelkens, C. 1998, A\&A, 331, 211

Millan-Gabet, R., Schloerb, F. P., \& Traub, W. A. 2001, ApJ, 546,358

Miroshnichenko, A. S., \& Corporon, P. 1999, A\&A, 349, 126

Miroshnichenko, A. S., Ivezić, Ž., Vinković, D., \& Elitzur, M. 1999, ApJ, 520, L115

Miroshnichenko, A. S., Chentsov, E. L., Klochkova, V. G., et al. 2000, A\&AS, 147, 5

Miroshnichenko, A. S., \& Bjorkman, K. S. 2000, in The Bephenomenon in Early-Type Stars, ed. M. A. Smith, H. F. Henrichs, \& J. Fabregat, ASP Conf. Ser., 214, 484

Neckel, Th., \& Klare, G. 1980, A\&AS, 42, 251

Oudmaijer, R. D., van der Ween, V. E. C. J., Waters, L. B. F. M., et al. 1992, A\&AS, 92, 625

Palla, F., \& Stahler, S. W. 1993, ApJ, 418, 414

Savage, B. D., \& Mathis, J. 1979, ARA\&A, 17, 73

Schaerer, D., Charbonnel, C., Meynet, G., Maeder, A., \& Schaller, G. 1993, A\&AS, 102, 339

Sheikina, T. A., Miroshnichenko, A. S., \& Corporon, P. 2000, in The Be-phenomenon in Early-Type Stars, ed. M. A. Smith, H. F. Henrichs, \& J. Fabregat, ASP Conf. Ser., 214, 494

Schild, R. E., Garrison, R. F., \& Hiltner, W. A. 1983, ApJS, 51,321

Thé, P. S., de Winter, D., \& Pérez, M. R. 1994, A\&AS, 104, 315

Volk, K., Kwok, S., Stencel, R. E., \& Brugel, E. 1991, ApJS, 77,607

Yudin, R. V., \& Evans, A. 1998, A\&AS, 131, 401

Zickgraf, F.-J., Wolf, B., Stahl, O., et al. 1986, A\&A, 163, 119 